\title{
Comments: "The use of high-resolution MRI to detect thrombosis and lipid-rich carotid artery plaques in a patient with homozygous familial hypercholesterolemia: a case report"
}

\author{
Dingting $\mid i^{1}$ \\ (iD) Xu Zhang ${ }^{1}$ \\ (iD) Baohong Xue \\ (iD) Lianping $\mathrm{He}^{1}$
}

1. College of experience industry, Anhui polytechnic university, Wuhu, Anhui 241000 , China. Telephone and fax: +86 553-2871231 Email: baohongxue@yeah.net

Dear Editor,We read with great interest the study by Wang et al. ${ }^{1}$ in which they demonstrated that high-resolution multi-contrast MRI played an excellent role in identifying carotid plaque components in a patient with homozygous familial hypercholesterolemia (HoFH). The authors indicate that data on carotid plaque burden may provide some information on patients with HoFH. In our opinion, we should combine a large number of relevant clinical cases to verify this.

Firstly, it is novel and innovative to use high-resolution MRI testing for plaque histology of patients with HoFH. Although the diagnostic accuracy of MRI is superior to CT in detecting thymomas, there is only one case included in present study ${ }^{2}$. Thus, more patients with HoFH should be recruited in future studies.
Secondly, high-resolution MRI has the ability to qualitatively and quantitatively evaluate the main components of advanced carotid atherosclerotic plaques. However, familial hypercholesterolemia is often perceived and described as underdiagnosed and undertreated $^{3}$. In this kind of patient with major symptoms, CT would be treated directly with endarterectomy, with no change of the treatment given by the MRI.

\section{REFERENCES}

1. Wang $Z$, Liu W, Jiang $L$, Wang $L$, Yu W. The use of high-resolution MRI to detect thrombosis and lipid-rich carotid artery plaques in a patient with homozygous familial hypercholesterolemia: a case report. Rev Assoc Med Bras. 2020;66 (1) 31-35.

2. Li HR, Gao J, Jin C, Jiang JH, Ding JY. Comparison between CT and MRI in the diagnostic accuracy of thymic masses. J Cancer. 2019;10(14):3208-13.

3. Ceska R, Latkovskis G, Ezhov MV, Freiberger T, Lalic K, Mitchenko O, et al. The impact of the international cooperation on familial hypercholesterolemia screening and treatment: results from the ScreenPro FH Project. Curr Atheroscler Rep. 2019;21(9):36. 